UWThPh-1998-6

March, 1998

\title{
Dirac Variables and Zero Modes of Gauss Constraint in Finite-Volume Two-Dimensional QED
}

\author{
S. Gogilidze ${ }^{a}$ \\ Institute for High Ehergy Physics \\ University of Tbilisi \\ Chavchavadze 3, 080 Tbilisi, Georgia \\ Nevena Ilieva ${ }^{*, b}$ \\ Institute for Theoretical Physics \\ University of Vienna \\ Boltzmanngasse 5, 1090 Vienna, Austria \\ V.N.Pervushin ${ }^{c}$ \\ Bogoliubov Laboratory of Theoretical Physics \\ Joint Institute for Nuclear Research \\ 141980 Dubna, Moscow Region, Russia
}

\begin{abstract}
The finite-volume $\mathrm{QED}_{1+1}$ is formulated in terms of Dirac variables by an explicit solution of the Gauss constraint with possible nontrivial boundary conditions taken into account. The intrinsic nontrivial topology of the gauge group is thus revealed together with its zero-mode residual dynamics. Topologically nontrivial gauge transformations generate collective excitations of the gauge field above Coleman's ground state, that are completely decoupled from local dynamics, the latter being equivalent to a free massive scalar field theory.

* On leave from Institute for Nuclear Research and Nuclear Energy, Bulgarian Academy of Sciences, Bul.Tzarigradsko Chaussee 72, 1784 Sofia, Bulgaria

${ }^{a}$ E-mail address: soso@jinr.ru

${ }^{b}$ E-mail address: ilieva@pap.univie.ac.at

${ }^{c}$ E-mail address: pervush@thsun1.jinr.ru
\end{abstract}




\section{Introduction}

Separation of real dynamical variables from nondynamical ones is the crucial step in extracting the relevant physical information from the gauge theories. The usual method for achieving this purpose - by imposing a gauge-fixing condition, might not be always adequate to the dynamical content of the classical equations of motion. Another possibility, offered by Dirac [1], consists in introduction of gauge invariant dynamical variables through an explicit solution of the Gauss equation. Such an explicit solution might contain some additional physical information which is implicitly lost by the gauge fixing. This can be seen even on the simplest example of the two-dimensional QED. It is our aim in the present paper to construct the Dirac variables for this model and to clarify the physical consequences from such an approach.

We first solve the classical equations for the free electromagnetic field in a finite volume to obtain the residual dynamics on the Gauss constraint. We show that this dynamics could not be reproduced by an arbitrary gauge choice.

Then we examplify the physical consequences of the existence of such a residual dynamics by the Schwinger model.

\section{Free Electromagnetic Field in a Finite Volume}

Let us start with the free Abelian gauge field in a finite-volume two-dimensional spacetime:

$$
\begin{aligned}
S & =\int_{-T / 2}^{T / 2} d t \int_{-R / 2}^{R / 2} d x \mathcal{L}_{0}(t, x) \\
\mathcal{L}_{0}(t, x) & =-\frac{1}{4} F^{\mu \nu}(t, x) F_{\mu \nu}(t, x),
\end{aligned}
$$

where $F_{\mu \nu}$ is the field-strenght tensor.

From this action, equations of motion follow

$$
\partial^{\mu} F_{\mu \nu}(t, x)=0
$$

the one for $\nu=0$ being actually a constraint:

$$
\partial_{1} \partial_{0} A_{1}(t, x)=\partial_{1}^{2} A_{0}(t, x) .
$$

This equation can be explicitly integrated, thus yielding the solution

$$
\begin{aligned}
A_{0}(t, x) & =\frac{1}{2}\left[A_{0}(t, R / 2)+A_{0}(t,-R / 2)\right]+\frac{x}{R}\left[A_{0}(t, R / 2)-A_{0}(t,-R / 2)\right]+ \\
& +\int_{-R / 2}^{R / 2} \mathcal{D}(x, y) \partial_{y} \dot{A}_{1}(t, y) d y
\end{aligned}
$$


where $\mathcal{D}(x, y)$ is the Green function for D'Alembert equation with zero boundary conditions

$$
\begin{gathered}
\mathcal{D}(x, y)=\mathcal{D}(y, x) \\
\mathcal{D}( \pm R / 2, y)=0, \quad \partial_{y} \mathcal{D}( \pm R / 2, y)=0
\end{gathered}
$$

Explicitly,

$$
\mathcal{D}(x, y)=\frac{|x-y|}{2}+\frac{x y}{R}-\frac{R}{4}
$$

so that

$$
\partial_{x} \partial_{y} \mathcal{D}(x, y)=-\delta(x-y)+\frac{1}{R} .
$$

We restrict ourselves to the case when $A_{0}(t, x)$ satisfies symmetric boundary conditions in $x$

$$
A_{0}(t,-R / 2)=A_{0}(t, R / 2)
$$

However, the $U(1)$ gauge invariance of the action (1)

$$
A_{\mu}(t, x) \rightarrow A_{\mu}^{g}(t, x)=A_{\mu}(t, x)+\frac{i}{e} \partial_{\mu} \lambda(t, x)
$$

with $\lambda(t, x)$ an arbitrary function, means that the same property takes place also for the gauge transformations parameter $\dot{\lambda}(t, x)$. Then, the $x$-derivative of $A_{0}(4)$ with eqs. (5), (7) taken into account becomes

$$
\begin{aligned}
\partial_{x} A_{0}(t, x) & =\int_{-R / 2}^{R / 2} \partial_{x} \mathcal{D}(x, y) \partial_{y} \dot{A}_{1}(t, y) d y= \\
& =\dot{A}_{1}(t, x)-\frac{1}{R} \int_{-R / 2}^{R / 2} \dot{A}_{1}(t, y) d y
\end{aligned}
$$

Thus, for the (only one) field-stregth tensor component we get

$$
\left.F_{01}(t, x)\right|_{\frac{\delta S}{\delta A_{0}}=0}=\frac{1}{R} \int_{-R / 2}^{R / 2} \dot{A}_{1}(t, y) d y=\bar{F}_{01}(t) .
$$

It is convenient to introduce a new collective variable

$$
N(t)=\frac{e}{2 \pi} \int_{-R / 2}^{R / 2} A_{1}(t, x) d x
$$

so that

$$
\bar{F}_{01}(t)=\frac{1}{R}\left(\frac{2 \pi}{e}\right) \dot{N}(t)
$$


In terms of $N(t)$ the free action (1) reads

$$
S^{T}=\frac{1}{2 R}\left(\frac{2 \pi}{e}\right)^{2} \int_{-T / 2}^{T / 2} \dot{N}^{2}(t) d t
$$

The remaining dynamical equation - eq.(2) for $\nu=1$, reduces to

$$
\ddot{N}(t)=0
$$

which also follows from action (13). The collective variable $N(t)$ (11) can be cosidered as a continuous generalization of the Pontryagin index $\nu$,

$$
\nu=\frac{e}{4 \pi} \int_{-T / 2}^{T / 2} d t \int_{-R / 2}^{R / 2} d x \epsilon_{\mu \nu} F^{\mu \nu}(t, x)=\int_{-T / 2}^{T / 2} d t \dot{N}(t)=N(T / 2)-N(-T / 2) .
$$

As a functional on $A(t, x)$, this variable is invariant under gauge transformations (9), supposed the boundary condition (8) is simply applied to the gauge function $\lambda(t, x)$, thus resulting in

$$
\dot{\lambda}(t,-R / 2)=\dot{\lambda}(t, R / 2) .
$$

\section{Quantum Dynamics of the Collective Variable}

The action so obtained, eq.(13), is relativistically invariant and can be straightforward quantized:

$$
[N, P]=i, \quad P=\frac{1}{R}\left(\frac{2 \pi}{e}\right)^{2} \dot{N}
$$

Thus, the gaugeless reduction of the action for the free Abelian gauge field results in a residual (actually longitudinal) action, describing the collective motion of the latter. This superfluid behaviour gives a deep insight into the interplay between the local dynamics and the global symmetries of the theory though could hardly be recovered in an arbitrary gauge.

The solution of corresponding Schrödinger equation, invariant under (9), is of the form of a plane wave with respect to $N$

$$
\Psi=e^{-i P N[A]}, \quad P=\frac{2 \pi}{e} E .
$$

Such an unsatisfactory result originates in the formal treatment of the boundary conditions for the problem. As is easily seen, both the variable (11) and the action (13) have no consistent classical interpretation. Therefore, when gauge transformations are to be considered as transformations of the quantum theory, the boundary conditions should be imposed on the gauge phase $e^{i \lambda(t, x)}$. In such a way, for the case at hand one has

$$
e^{i \lambda(R / 2)}=e^{i \lambda(-R / 2)}
$$


that is

$$
\lambda(R / 2)-\lambda(-R / 2)=2 \pi n, \quad n \in \mathbf{Z}
$$

Thus, a mapping of the (finite) space $R(1)$ onto the group manifold of $U(1)$ is defined, with a (possibly) nontrivial degree of mapping $n$. The gauge fields are so devided into topological classes, characterized by the value of $n$, their configuration space acquiring the topology of a ring. However, points with different $n$ are physically equivalent, so that the wave function of the gauge field in them may differ only by a phase factor

$$
\Psi[N+1]=e^{i \theta} \Psi[N], \quad|\theta|<\pi
$$

In such a theory, a purely quantum phenomenon - a field Josephson effect, takes place. Due to the inhomogeneity of the wave function phase, eq.(3.5), a nonvanishing electric field without any sources is present in the ground state [2, 3].

Indeed, now the collective variable $N[A]$ is only covariant under (9),(17) - so, under topologically nontrivial or large gauge transformations,

$$
N\left[A^{g}(t, x)\right]=N[A(t, x)]+n
$$

and the spectrum of its conjugate momentum $P$ is essentially changed due to the boundary condition (18). It indicates the equivalence of the states

$$
\langle P \mid N\rangle=e^{i P N} \quad \text { and } \quad\langle P \mid N+n\rangle=e^{i P(N+n)},
$$

the true state thus representing a Bloch wave

$$
\begin{array}{r}
\langle P \mid N\rangle=\lim _{l \rightarrow \infty} \frac{1}{l} \sum_{n=-l / 2}^{l / 2} e^{i n \theta} e^{-i P(N+n)}= \\
=\left\{\begin{array}{cc}
e^{-i N(2 \pi k+\theta)}, & P=2 \pi k+\theta \\
0, & P \neq 2 \pi k+\theta,
\end{array}\right. \\
k \in \mathbf{Z}, \quad|\theta|<\pi
\end{array}
$$

This means that the operator $\hat{E}$ describes a constant electric field and its spectrum is

$$
\hat{E} \Psi=\frac{e}{2 \pi} P \Psi=e\left(k+\frac{\theta}{2 \pi}\right) \Psi
$$

So, we have obtained excitations above Coleman's ground state [4, which corresponds to the minimal (in modulus) eigenvalue of the operator $\hat{E}$ (for $k=0$ ). However, the interpretation is essentially different: while in [4] the existence of $\theta$, as an additional parameter, is justified by the properties of the space $R(1)$, here it appears as a bridge between the global symmetries and the local dynamics of the theory. Moreover, consideration of $\theta$ as an additional (continuous) parameter implies already a non-separable Hilbert space due to the existence of an uncountable set of orthogonal vectors (corresponding to different values of $\theta$ ), with all complications that follow from this. 
It should be emphasized that this is an entirely quantum effect. The residual topological action (13), as already mentioned, has no consistent classical interpretation, since the region of "validity" of the quantum theory is proportional to the volume $R$ of the space. Therefore, in consideration of an infinite-volume model, the infrared regularization should be removed at the very final stage and only the final finite-volume results have to be checked for surviving the thermodynamic limit. An illustration of the importance of this observation is offered by the charge screening in the Schwinger model [5].

\section{Hamiltonian dynamics in the first order formalism}

As has been mentioned in Sec.3, the global excitation of the gauge field could be easily overlooked by an unappropriate gauge choice. However, this is not necessarily so, as shows consideration of the free action (1) in the first order formalism:

$$
S_{I}\left[E, A^{\mu}\right]=\int_{-T / 2}^{T / 2} d t \int_{-R / 2}^{R / 2} d x\left[E\left(\dot{A}_{1}-\partial A_{0}\right)-(1 / 2) E^{2}\right] .
$$

The canionical momenta define the primary constraints

$$
\phi_{1}=\pi_{E}=0, \quad \phi_{2}=\pi_{1}-E=0, \quad \phi_{3}=\pi_{0}=0 .
$$

Thus the total Hamiltonian takes the form

$$
H_{T}(t)=\int_{-R / 2}^{R / 2} d x\left[(1 / 2) E^{2}+E \partial A_{0}+u_{1} \pi_{E}+u_{2}\left(\pi_{1}-E\right)+u_{3} \pi_{0}\right] .
$$

Among the primary constraints there are one first class constraint and two second class ones

$$
\begin{gathered}
\left\{\phi_{1}, \phi_{2}\right\}=\delta\left(x-x^{\prime}\right) \\
\left\{\phi_{1}, \phi_{3}\right\}=\left\{\phi_{2}, \phi_{3}\right\}=0
\end{gathered}
$$

The stationarity conditions for the primary constraints fix two Lagrange multipliers

$$
u_{1}=0 \quad u_{2}=E-\partial A_{0}
$$

and generate one secondary constraint

$$
\Phi=\partial E=0
$$

which is itself stationary so that no more constraints appear in the theory.

With the following admissible gauge-fixing conditions:

$$
\chi_{1}=A_{0}=0 \quad \chi_{2}=\pi_{E}=0
$$


the final Hamiltonian is obtained

$$
H(t)=\int_{-R / 2}^{R / 2} d x\left[(1 / 2) E^{2}+E \partial A_{0}++\left(E-\partial A_{0}\right)\left(\pi_{1}-E\right)\right] .
$$

Thus the Hamiltonian equations of motion are obtained to be

$$
\begin{gathered}
\dot{A}_{0}=0, \quad \dot{\pi}_{0}=0, \quad \dot{A}_{1}=E-\partial A_{0}, \quad \dot{\pi}_{1}=0, \quad \dot{E}=0, \quad \dot{\pi}_{E}=0, \\
\pi_{E}=0, \quad \pi_{1}-E=0, \quad \pi_{0}=0, \quad \partial E=0, \quad A_{0}=0 .
\end{gathered}
$$

The solution of this system is

$$
E(x, t)=\frac{e}{2 \pi} P(t)
$$

and depending on the boundary conditions, reproduces our previous results.

\section{Dirac Observables for the Finite-Volume QED $_{1+1}$}

The finite--volume Schwinger model is characterized by the action

$$
S=\int_{-T / 2}^{T / 2} d t \int_{-R / 2}^{R / 2} d x \mathcal{L}(\underline{x})
$$

with Lagrangean density

$$
\begin{gathered}
\mathcal{L}(\underline{x})=-\frac{1}{4} F^{\mu \nu}(\underline{x}) F_{\mu \nu}(\underline{x})+i \bar{\psi}(\underline{x}) \gamma_{\mu}\left(\partial^{\mu}-i e A^{\mu}(\underline{x})\right) \psi(\underline{x}), \\
\underline{x}=(t, x), \quad \operatorname{diag} g_{\mu \nu}=(1,-1)
\end{gathered}
$$

where $\psi(x), \bar{\psi}(x)$ are anticommuting two-component spinors and the two-dimensional $\gamma$-matrices are

$$
\gamma_{0}=\sigma_{1}, \quad \gamma_{1}=-i \sigma_{2}, \quad \gamma_{5}=\gamma_{0} \gamma_{1}=\sigma_{3}
$$

with $\sigma_{k}$ being the Pauli matrices.

Equations of motion now read

$$
\begin{gathered}
-\partial^{\mu} F_{\mu \nu}(\underline{x})=J_{\nu}(\underline{x}), \quad J_{\mu}(\underline{x})=\bar{\psi}(\underline{x}) \gamma_{\mu} \psi(\underline{x}) \\
\gamma_{\mu}\left(\partial^{\mu}-i e A^{\mu}(\underline{x})\right) \psi(\underline{x})=0
\end{gathered}
$$

so that the constraint - eq.(25a) for $\nu=0$, depends also on the current

$$
\partial_{1}^{2} A_{0}(\underline{x})=\partial_{1} \partial_{0} A_{1}(\underline{x})+J_{0}(\underline{x})
$$


An explicit solution of the constraint equation can still be obtained

$$
\begin{aligned}
A_{0}(t, x) & =\frac{1}{2}\left[A_{0}(t, R / 2)+A_{0}(t,-R / 2)\right]+\frac{x}{R}\left[A_{0}(t, R / 2)-A_{0}(t,-R / 2)\right]+ \\
& +\int_{-R / 2}^{R / 2} \mathcal{D}(x, y)\left[\partial_{y} \dot{A}_{1}(\underline{y})+J_{0}(\underline{y})\right] d y
\end{aligned}
$$

with $\mathcal{D}(x, y)$ given by $(5),(6)$.

On the solution (27), the field-strenght tensor takes the form

$$
\begin{aligned}
\left.F_{01}(t, x)\right|_{\frac{\delta S}{\delta A_{0}}=0} & =\frac{1}{R} \int_{-R / 2}^{R / 2} \dot{A}_{1}(t, y) d y+\int_{-R / 2}^{R / 2}\left(\frac{1}{2} \epsilon(y-x)-\frac{y}{R}\right) J_{0}(\underline{y}) d y \\
& =\bar{F}_{01}(t)-\bar{Q}+\frac{1}{2} \int_{-R / 2}^{R / 2} \epsilon(y-x) J_{0}(\underline{y}) d y
\end{aligned}
$$

with the charge $\bar{Q}$ defined as

$$
\bar{Q}=\frac{1}{R} \int_{-R / 2}^{R / 2} x J_{0}(\underline{x}) d x
$$

Following the procedure proposed by Dirac [1], we introduce now a gauge invariant set of variables - the Dirac observables. This is achieved by a gauge transformation

$$
A_{\mu}^{D}(\underline{x})=A_{\mu}(\underline{x})-\frac{\partial_{\mu} \lambda^{D}(\underline{x})}{e}, \quad \psi^{D}(\underline{x})=e^{-i \lambda^{D}(\underline{x})} \psi(\underline{x})
$$

with a gauge parameter

$$
\dot{\lambda}^{D}(\underline{x})=\frac{e}{2} \int_{-R / 2}^{R / 2} \epsilon(x-y) \dot{A}_{1}(\underline{y}) d y+\frac{e}{2}\left[A_{0}(t, R / 2)+A_{0}(t,-R / 2)\right]
$$

which gives for the gauge field components

$$
\begin{aligned}
& A_{1}^{D}(\underline{x})=0 \\
& A_{0}^{D}(\underline{x})=-x \bar{F}_{01}(t)+\int_{-R / 2}^{R / 2} \mathcal{D}(x, y) J_{0}(\underline{y}) d y
\end{aligned}
$$

With (28) in mind, the reduced Lagrangean can be written, entirely in terms of observables (30), (32):

$$
\begin{aligned}
L & =i \bar{\psi}^{D} \gamma_{\mu} \partial^{\mu} \psi^{D}+\frac{1}{2 R}\left(\frac{2 \pi}{e}\right)^{2} \dot{N}^{2}-2\left(\frac{2 \pi}{e}\right) \dot{N} \bar{Q}+ \\
& +\frac{1}{2} \int_{-R / 2}^{R / 2} J_{0}(\underline{x}) \mathcal{D}(x, y) J_{0}(\underline{y}) d x d y
\end{aligned}
$$


Here, relation (12) has also been used in order to get the collective variable $N[A]$ explicitly entering the reduced action, so that the interaction between the local fields and the global collective mode becomes apparent.

\section{Currents, Symmetries and Charge Conservation in Finite Volume}

As is well known, the current commutator in two dimensions is afflicted with an anomaly — the so-called Schwinger term,

$$
\left[J_{0}(x), J_{1}(y)\right]=\frac{i e^{2}}{\pi} \delta^{\prime}(x-y),
$$

which is just a manifestation of the Dirac vacuum needed for a consistent quantum theory to be written, even in the case of free fermions, but also when various types of interactions are present (see, for example [6, 7, 2], also [8] for the 4-dimensional case).

Relation (34) is the ground for the bosonization in two dimensions, which is formally achieved (for fermion bilinears) through the following identification

$$
J_{5 \mu}(x)=\frac{e}{\sqrt{\pi}} \partial_{\mu} \phi(x), \quad J_{5 \mu}=\bar{\psi}(x) \gamma_{5} \gamma_{\mu} \psi(x)
$$

Thus, from Lagrangean (33) the following reduced Hamiltonian is obtained

$$
\begin{aligned}
H^{r e d} & =\frac{1}{2} R\left(\frac{e}{2 \pi}\right)^{2}\left(P_{N}+\frac{4 \pi}{e} \bar{Q}\right)^{2}-\frac{e^{2}}{2 \pi} R \bar{\phi}^{2}+ \\
& +\frac{1}{2} \int_{-R / 2}^{R / 2}\left[\Pi^{2}(x)+\left(\partial_{1} \phi(x)\right)^{2}+\frac{e^{2}}{\pi} \phi^{2}(x)\right] d x
\end{aligned}
$$

where $\Pi(x)$ is the field- $\phi$ conjugate momentum

$$
[\phi(x), \Pi(y)]=i \delta(x-y), \quad \Pi(x) \equiv \partial_{0} \phi(x)=\frac{\sqrt{\pi}}{e} J_{50}(x),
$$

$P_{N}$ is the topological momentum

$$
P_{N}=\frac{\partial L}{\partial \dot{N}}=\frac{1}{R}\left(\frac{2 \pi}{e}\right)^{2} \dot{N}-\frac{4 \pi}{e} \bar{Q}, \quad\left[N, P_{N}\right]=i
$$

and the "mean field" $\bar{\phi}$ is defined as

$$
\bar{\phi}=\frac{1}{R} \int_{-R / 2}^{R / 2} \phi(x) d x
$$


The second term in the r.h.s. of eq.(36) has its origin in the finiteness of the space, hence in the specific form of the Green function $\mathcal{D}(x, y)$ (see eqs.(6), (7)). Clearly, it vanishes in the limit $R \rightarrow \infty$, but we have already emphasized that the level of Hamiltonian is an early one for taking this limit. In what follows, we will specially underline the effects that are brought to being through this term.

In the model under consideration, despite the usual electric and chiral charges

$$
\begin{aligned}
Q_{0} & =\int_{-R / 2}^{R / 2} J_{50}(x) d x=-\frac{e}{\sqrt{\pi}} \int_{-R / 2}^{R / 2} \partial_{1} \phi(x)=-\frac{e}{\sqrt{\pi}}[\phi(R / 2)-\phi(-R / 2)] \\
Q_{5} & =\int_{-R / 2}^{R / 2} J_{50}(x) d x=\frac{e}{\sqrt{\pi}} \int_{-R / 2}^{R / 2} \Pi(x) d x
\end{aligned}
$$

also the charge $\bar{Q}(29)$ can be constructed, which is related to the field $\bar{\phi}$ through the equivalence relations $(35)$

$$
\bar{Q}=\frac{e}{R \sqrt{\pi}} \int_{-R / 2}^{R / 2} x \partial_{1} \phi(x) d x=\frac{e}{\sqrt{\pi}}\left[\bar{\phi}+(1 / 2) Q_{0}\right] .
$$

The zero-charge sector corresponds to a symmetric boundary condition on $\phi(x)$,

$$
\phi(R / 2)=\phi(-R / 2),
$$

in this case the charge $\bar{Q}$ is simply proportional to $\bar{\phi}$ and vanishes together with it in the limit $R \rightarrow \infty$. At finite $R$, it does not correspond to an invariance of the theory

$$
e^{i \alpha \bar{Q}} H^{r e d} e^{-i \alpha \bar{Q}}=H^{r e d}-\alpha Q_{5} / R,
$$

but this is also the case in the original fermionic formulation. The situation with the chiral charge, hence with the (global) chiral symmetry, is more subbtle: chiral transformations, implemented by $\sigma=\exp \left\{i \sqrt{\pi} Q_{5} / e\right\}$, do correspond to an invariance of the original fermionic action (1). The descendent of the fermions - field $\phi(x)$, is itself not invariant but instead is shifted by an integer

$$
e^{i n Q_{5} \sqrt{\pi} / e} \phi(x) e^{-i n Q_{5} \sqrt{\pi} / e}=\phi(x)+n, \quad n \in \mathbf{Z}
$$

The dynamics, due to the interplay between this local field and the collective mode $N$, is nevertheless chiral invariant and the charge $Q_{5}$ is conserved

$$
i\left[H^{r e d}, Q_{5}\right]=0
$$

Thus, there is no global chiral symmetry broken to worry about, so no ground for an $U(1)$ problem. It is worthwhile noticing that (42) results from cancellation of contributions, generated by the boundary term and the genuine mass term, and by vanishing 
of the contribution from the kinetic term alone. This would not be the case if we would have removed the infrared regularization already in the reduced Hamiltonian, since then there would be nothing to compensate the mass-term contribution and the global chiral symmetry would have been broken.

The local chiral current, however, remains anomalous,

$$
\begin{aligned}
\partial^{\mu} J_{5 \mu} & =\left(\frac{e}{\sqrt{\pi}}\right)^{3}(\phi(x)-\bar{\phi})- \\
& -\frac{e}{\sqrt{\pi}}\left[\phi^{\prime}(R / 2) \delta(R / 2-x)-\phi^{\prime}(-R / 2) \delta(R / 2+x)\right] .
\end{aligned}
$$

With the reasonable assumption

$$
\phi^{\prime}(R / 2)=\phi^{\prime}(-R / 2)
$$

and with (35) in mind, eq.(43) takes the form of the Klein-Gordon equation for a free massive scalar field

$$
\left(\square+m^{2}\right) \tilde{\phi}(x)=0, \quad m=e / \sqrt{\pi}
$$

This is not an equation for the field $\phi(x)$ itself, but for the field

$$
\tilde{\phi}(x)=\phi(x)-\bar{\phi}
$$

This should be expected since the Hamiltonian (36) can be represented also as

$$
\begin{aligned}
H^{r e d} & =\frac{1}{2} R\left(\frac{e}{2 \pi}\right)^{2} P_{N}^{2}+2 R \bar{Q}\left(\bar{Q}+\frac{e}{2 \pi} P_{N}\right)+ \\
& +\frac{1}{2} \int_{-R / 2}^{R / 2}\left[\Pi^{2}(x)+\left(\partial_{1} \tilde{\phi}(x)\right)^{2}+\frac{e^{2}}{\pi} \tilde{\phi}^{2}(x)\right] d x
\end{aligned}
$$

which makes (44) apparent.

There is one special case,

$$
\bar{\phi}=-\frac{P_{N}}{2 \sqrt{\pi}}
$$

so then $\tilde{\phi}(x)$ appears as a combined field 円

$$
\tilde{\phi}_{s}(x)=\phi(x)+\frac{P_{N}}{2 \sqrt{\pi}}
$$

In this case there is no explicit interaction term, though the interaction between the global mode $N$ and the local field $\tilde{\phi}(x)$ is intrinsic due to the very structure of the latter. Now the chiral charge is no longer conserved

$$
\dot{Q}_{5}=m^{3} R P_{N} / \sqrt{\pi}
$$

\footnotetext{
${ }^{1}$ Still one has to think about the precise meaning of this field in the context of (21)
} 
and the axial current, still being anomalous, does not provide a free equation for $\tilde{\phi}(x)$ but either an interacting one for the latter

$$
\left(\square+m^{2}\right) \tilde{\phi}(x)=m^{2} \frac{p_{N}}{2 \sqrt{\pi}}
$$

or a free equation but for the original field $\phi(x)$

$$
\left(\square+m^{2}\right)(\tilde{\phi}(x)+\bar{\phi})=0 .
$$

However, the action is invariant under simultaneous transformations of both fields

$$
\tilde{\sigma}^{n} H^{r e d}\left[\tilde{\phi}_{s}\right] \tilde{\sigma}^{-n}=H^{r e d}\left[\tilde{\phi}_{s}\right], \quad \tilde{\sigma}=e^{i \sqrt{\pi}\left(Q_{5}-2 e N\right) / e}=e^{i \sqrt{\pi} \tilde{Q}_{5} / e}
$$

which have to be considered as the new chiral transformations. The charge $\tilde{Q}_{5}$ is conserved but no local current can be associated with it.

\section{Concluding Remarks}

We have examplified by two simple models the advantages of the application of Dirac variables for quantization of gauge theories. Introduction of these variables based on the explicit solution of the constraint equations, provides some additional dynamical information, which is implicitly lost by the gauge fixing.

Thus, already in the case of a free two-dimensional electromagnetic field in a finite volume, the zero-mode sector shows a superfluid behaviour, due to the residual topological dynamics of the Abelian gauge field, which however survives in the thermodynamic limit. It is the existence of this global (topological) mode that is responcible for the appearance of the $\theta$-vacuum, which is therefore present also in the free theory.

Consideration of the finite-volume Schwinger model in the same spirit leads to an unexpected result, namely the conservation of the chiral charge, though the local chiral current remains anomalous. This is now only a reason for the mass generation and not an explanation for the absence of the Nambu-Goldstone phenomenon, since the latter has simply no ground in this case. The $\theta$-vacuum structure is nevertheless justified also in the finite-volume considerations. Thus, the model examplifies the independence between the global chiral symmetry (its breaking and restoration) and the nontrivial vacuum structure, underlying the purely topological origin of the latter.

Though the global mode appears to be formally decoupled from local dynamics, its contribution is crucial for the symmetry properties of the model. This shows once again the severe influence of the spurious deggrees of freedom on the physical interpretation of the quantized constraint dynamics.

\section{Acknowledgements}

It is a pleasant duty to thank R. Bertlmann, V.A. Efremov, A.M. Khvedelidze, E.A. Kuraev and $\mathrm{W}$. Thirring for stimulating discussions. We acknowledge hospitality at the 
Institute for Theoretical Physics of Vienna University, where this paper has been completed.

This work has been supported in part by the "Fonds zur Förderung der wissenschaftlichen Forschung in Österreich", Project P11287-PHY, and by the Russian Foundation for Basic Investigation, Grant No. 96-01-01223.

\section{References}

[1] P.A.M. Dirac, Can. J. Phys. 33, 650 (1955).

[2] N. Ilieva and V.N. Pervushin, Sov. J. Nucl. Phys. 39, 638 (1984).

[3] V.N. Pervushin, Riv. Nuovo Cim. 8, No.10, 1 (1985).

[4] S. Coleman, Ann. Phys. (N.Y.) 101, 239 (1976).

[5] N. Ilieva and V.N. Pervushin, Int. J. Mod. Phys. A4, 4567 (1989).

[6] P. Jordan, Z. Phys. 93, 464 (1935); 98, 759 (1936); 99, 109 (1936); 102, 243 (1936); 105, 229 (1937).

[7] A. Mattis, E. Lieb, J. Math. Phys. 6, 304 (1965).

[8] V.N. Gribov, Nucl. Phys. B139, 1 (1978). 